\title{
Two Component Plasma in a Flamm's Paraboloid
}

\author{
Riccardo Fantoni \\ E-mail: rfantoni27@sun.ac.za \\ National Institute for Theoretical Physics (NITheP) and Institute of Theoretical \\ Physics, University of Stellenbosch, Stellenbosch 7600, South Africa
}

\begin{abstract}
The two component plasma (TCP) living in a Flamm's paraboloid is studied at a value of the coupling constant $\Gamma=2$ for which an analytic expression for the grand canonical partition function is available. Two cases are considered, the plasma in the half surface with an insulating horizon and the plasma in the whole surface. The Green's function equation necessary to determine the $n$-particle truncated correlation functions is explicitly found. In both cases this proves too complicated to be solved analytically. So we present the method of solution reducing the problem to finding the two linearly independent solutions of a linear homogeneous second order ordinary differential equation with polynomial coefficients of high degrees. In the flat limit one recovers the solution for the structure of the TCP in a plane in the first case but the collapse of opposite charges at the horizon makes the structure of the plasma physically not well defined in the second case.
\end{abstract}

PACS numbers: 05.20.Jj,52.27.Cm,52.27.Gr,68.15.+e 


\section{Introduction}

The two component plasma (TCP) is a neutral mixture of point wise particles of charge $\pm q$. The equation of state of the TCP living in a plane is known since the work of Salzberg and Prager [1]. In the plasma the attraction between oppositely charged particles competes with the thermal motion and makes the partition function of the finite system diverge when $\Gamma=\beta q^{2} \geq 2$, where $\beta=1 / k_{B} T$ with $k_{B}$ Boltzmann constant. The system becomes unstable against the collapse of pairs of oppositely charged particles, and as a consequence all thermodynamic quantities diverge, so that the point particle model is well behaved only for $\Gamma<2[2]$ when the Boltzmann factor for unlike particles is integrable at small separations of the charges. In this case rescaling the particles coordinates so as to stay in the unit disk one easily proves that the grand canonical partition function is a function of $\sqrt{\lambda_{-} \lambda_{+}} V^{(1-\Gamma / 4)}$, where $V$ is the volume of the plasma and $\lambda_{ \pm}$the fugacities of the two charge species, and as a consequence the equation of state is $\beta P=n(1-\Gamma / 4)$ where $n=n_{+}+n_{-}$is the total particle number density. It also follows that the ratio $\sqrt{\lambda_{-} \lambda_{+}} / n^{(1-\Gamma / 4)}$ must be a function of $\Gamma$ only in the thermodynamic limit [3]. However, if the collapse is avoided by some short range repulsion (hard cores for instance), the model remains well defined for lower temperatures. Then, for $\Gamma>4$ the long range Coulomb attraction binds positive and negative particles in pairs of finite polarizability. Thus, at some critical value $\Gamma_{c} \sim 4$ the system undergoes the Kosterlitz-Thouless transition [4] between a high temperature $(\Gamma<4)$ conductive phase and a low temperature $(\Gamma>4)$ dielectric phase. The same behavior also occurs in the TCP living in one dimension [5].

The structure of the TCP living in a plane at the special value $\Gamma=2$ of the coupling constant is solvable exactly analytically [6]. Through the use of an external potential it has also been studied in various confined geometries [7, 8, 9, 10] and in a gravitational field [11, 12].

On curved surfaces it has only been studied in surfaces of constant curvature as the sphere [13, 14] and the pseudosphere [15]. Unlike the one component plasma where the properties of the Vandermonde determinant allowed the analytical solution a Cauchy identity is used for the solution of the TCP. Unlike in the one component case where the solution was possible for the plasma confined in a region of the surface now this is not possible, anymore, without the use of an external potential. In these cases the external potential is rather given by $-\left(\Gamma / q^{2}\right) \ln \sqrt{g}$ where $g$ is the determinant of the metric tensor.

In this work we study the TCP at the special value $\Gamma=2$ of the coupling constant in a Flamm's paraboloid, a surface of non constant curvature. In this surface the one component plasma [16] has been studied in various situations: confined to one "universe" by an insulating or a grounded perfect conductor "horizon" or in the whole surface. Here we will restrict ourselves only to the first and last situation. 
On a curved surface, even though the finite system partition function will still be finite for $\Gamma<2$ since the surface is locally flat, the structure will change respect to the flat case. The purpose of the present work is to see how it changes for the special case $\Gamma=2$ where an exact analytical solution is possible.

\section{The Flamm's paraboloid $\mathcal{S}$}

In this work, we want to study a two dimensional TCP in the Flamm's paraboloid. This is a Riemannian surface $\mathcal{S}$ with the following metric

$$
d \mathbf{s}^{2}=g_{\mu \nu} d x^{\mu} d x^{\nu}=\left(1-\frac{2 M}{r}\right)^{-1} d r^{2}+r^{2} d \varphi^{2}
$$

or $g_{r r}=1 /(1-2 M / r), g_{\varphi \varphi}=r^{2}$, and $g_{r \varphi}=0$.

The Flamm's paraboloid is an embeddable surface in the three-dimensional Euclidean space. It is composed by two identical "universes": $\mathcal{S}_{+}$and $\mathcal{S}_{-}$. These are both multiply connected surfaces connected by a hole of radius $2 M$. We will from now on call the $r=2 M$ region of the surface its "horizon". The scalar curvature is $R=-2 M / r^{3}$.

The system of coordinates $(r, \varphi)$ with the metric (1) has the disadvantage that it requires two charts to cover the whole surface $\mathcal{S}$. It can be more convenient to use the variable

$$
u= \pm \sqrt{\frac{r}{2 M}-1}
$$

instead of $r$. This gives the following metric when using the system of coordinates $(u, \varphi)$,

$$
d \mathbf{s}^{2}=4 M^{2}\left(1+u^{2}\right)\left[4 d u^{2}+\left(1+u^{2}\right) d \varphi^{2}\right] .
$$

The region $u>0$ corresponds to $\mathcal{S}_{+}$and the region $u<0$ to $\mathcal{S}_{-}$.

The Laplace-Beltrami operator is

$$
\begin{aligned}
\Delta f & =\frac{1}{\sqrt{g}} \frac{\partial}{\partial q^{\mu}}\left(\sqrt{g} g^{\mu \nu} \frac{\partial}{\partial q^{\nu}}\right) f \\
& =\left[\left(1-\frac{2 M}{r}\right) \partial_{r}^{2}+\frac{1}{r^{2}} \partial_{\varphi}^{2}+\left(\frac{1}{r}-\frac{M}{r^{2}}\right) \partial_{r}\right] f,
\end{aligned}
$$

where $\mathbf{q} \equiv(r, \varphi)$. Finding the Green's function of the Laplacian, naturally [16] leads to consider the system of coordinates $(s, \varphi)$, with

$$
s=\left(\sqrt{u^{2}+1}+u\right)^{2} .
$$

The range for the variable $s$ is ]0, $+\infty$ [. The lower paraboloid $\mathcal{S}_{-}$corresponds to the region $0<s<1$ and the upper one $\mathcal{S}_{+}$to the region $s>1$. A point in the upper paraboloid with coordinate $(s, \varphi)$ has a mirror image by reflection $(u \rightarrow-u)$ in the lower paraboloid, with coordinates $(1 / s, \varphi)$, since if

$$
s=\left(\sqrt{u^{2}+1}+u\right)^{2}
$$


then

$$
\frac{1}{s}=\left(\sqrt{u^{2}+1}-u\right)^{2} .
$$

In the upper paraboloid $\mathcal{S}_{+}$, the new coordinate $s$ can be expressed in terms of the original one, $r$, as

$$
s=\frac{(\sqrt{r}+\sqrt{r-2 M})^{2}}{2 M} .
$$

Using this system of coordinates, the metric takes the form of a flat metric multiplied by a conformal factor

$$
d \mathbf{s}^{2}=\frac{M^{2}}{4}\left(1+\frac{1}{s}\right)^{4}\left(d s^{2}+s^{2} d \varphi^{2}\right) .
$$

The Laplacian also takes a simple form

$$
\Delta f=\frac{s}{\sqrt{g}} \Delta_{\text {flat }} f
$$

where

$$
\Delta_{\text {flat }} f=\partial_{s}^{2} f+\frac{1}{s} \partial_{s} f+\frac{1}{s^{2}} \partial_{\varphi}^{2} f
$$

is the Laplacian of the flat Euclidean space $\mathbb{R}^{2}$. The square root of the determinant of the metric is now given by $\sqrt{g}=(M / 2)^{2} s\left(1+s^{-1}\right)^{4}$.

It is useful to keep in mind the following small $M$ behaviors

$$
\begin{aligned}
s & =2 \frac{r}{M}-2-\frac{1}{2} \frac{M}{r}-\frac{1}{2}\left(\frac{M}{r}\right)^{2}-\frac{5}{8}\left(\frac{M}{r}\right)^{3}+O\left(M^{4}\right) \\
\sqrt{g} & =\frac{r M}{2}+\frac{M^{2}}{2}+\frac{5 M^{3}}{8 r}+O\left(M^{4}\right) .
\end{aligned}
$$

\section{Coulomb potential created by a point charge}

We here summarize the results found in In Ref. [16] on the determination of the Coulomb potential between two charges living in half Flamm's paraboloid with an insulating horizon and between two charges living in the full Flamm's paraboloid.

The Coulomb potential $G\left(s, \varphi ; s_{0}, \varphi_{0}\right)$ created at $(s, \varphi)$ by a unit charge at $\left(s_{0}, \varphi_{0}\right)$ is given by the Green's function of the Laplacian

$$
\Delta G\left(s, \varphi ; s_{0}, \varphi_{0}\right)=-2 \pi \delta^{(2)}\left(s, \varphi ; s_{0}, \varphi_{0}\right)
$$

with appropriate boundary conditions. The Dirac distribution is given by

$$
\delta^{(2)}\left(s, \varphi ; s_{0}, \varphi_{0}\right)=\frac{1}{\sqrt{g}} \delta\left(s-s_{0}\right) \delta\left(\varphi-\varphi_{0}\right)
$$

Notice that using the system of coordinates $(s, \varphi)$ the Laplacian Green's function equation takes the simple form

$$
\Delta_{\text {flat }} G\left(s, \varphi ; s_{0}, \varphi_{0}\right)=-2 \pi \frac{1}{s} \delta\left(s-s_{0}\right) \delta\left(\varphi-\varphi_{0}\right)
$$

which is formally the same Laplacian Green's function equation for a flat space. 
The Laplacian Green's function equation (14) can be solved, as usual, by using the decomposition as a Fourier series. Since Eq. (14) reduces to the flat Laplacian Green's function equation (16), the solution is the standard one

$$
G\left(s, \varphi ; s_{0}, \varphi_{0}\right)=\sum_{n=1}^{\infty} \frac{1}{n}\left(\frac{s_{<}}{s_{>}}\right)^{n} \cos \left[n\left(\varphi-\varphi_{0}\right)\right]+g_{0}\left(s, s_{0}\right)
$$

where $s_{>}=\max \left(s, s_{0}\right)$ and $s_{<}=\min \left(s, s_{0}\right)$.

We consider now the case of particles restricted to live in the half surface (hs) $\mathcal{S}_{+}, s>1$, by a hard wall located at the "horizon", $s=1$. The region $\mathcal{S}_{-}, s<1$, is empty and has the same dielectric constant as the upper region. We want to consider a model where the interaction potential reduces to the flat Coulomb potential in the flat limit $M \rightarrow 0$. The solution of the Laplacian Green's function equation is given in Fourier series by Eq. (17). The 0th order Fourier component $g_{0}$ can be determined by the requirement that, in the limit $M \rightarrow 0$, the solution reduces to the flat Coulomb potential

$$
G^{\text {flat }}\left(\mathbf{r}, \mathbf{r}^{\prime}\right)=-\ln \frac{\left|\mathbf{r}-\mathbf{r}^{\prime}\right|}{L}
$$

where $L$ is an arbitrary constant length. Recalling that $s \sim 2 r / M$, when $M \rightarrow 0$, we find

$$
g_{0}\left(s, s_{0}\right)=-\ln s_{>}-\ln \frac{M}{2 L}
$$

and in the half surface

$$
G^{\mathrm{hs}}\left(s, \varphi ; s_{0}, \varphi_{0}\right)=-\ln \left|z-z_{0}\right|-\ln \frac{M}{2 L},
$$

where we defined $z=s e^{i \varphi}$ and $z_{0}=s e^{i \varphi_{0}}$. And $\lim _{M \rightarrow 0} G^{\mathrm{hs}}=G^{\text {flat }}$ as desired.

A similar procedure [16] gives the Coulomb potential between charges living in the whole surface (ws) $0<s<\infty$,

$$
G^{\mathrm{ws}}\left(s, \varphi ; s_{0}, \varphi_{0}\right)=-\ln \frac{\left|z-z_{0}\right|}{\sqrt{\left|z z_{0}\right|}}-\ln \frac{L_{0}}{L},
$$

with $L_{0}$ another length scale.

\section{The two component plasma model}

The TCP is a neutral mixture of two species of point charges of charge $\pm q$. In this work we want to study the plasma on the Flamm's paraboloid. In Ref. [16] the Coulomb potential between two charges living in the whole paraboloid, on half surface with an insulating horizon, and in half surface with a grounded horizon was found. The first and last case have been summarized in the previous section as they will be the two cases considered next. 


\section{TCP in the half surface with an insulating horizon}

When the TCP lives in the half surface with an insulating horizon the Coulomb potential is given by Eq. (20). We will use $u_{i}=s_{i} e^{i \varphi_{i}}$ and $v_{j}=s_{j} e^{i \varphi_{j}}$ to denote the complex coordinates of the positively and negatively charged particles respectively, where $s=(\sqrt{r}+\sqrt{r-2 M})^{2} / 2 M>1$. Then, following Ref. [6], we use a Cauchy identity [17] to rewrite $e^{-\beta U_{2 N}}$, where $U_{2 N}$ is the potential energy of a neutral system of $N$ positive and $N$ negative charges, as follows

$$
\begin{aligned}
& \exp \left\{2 \sum_{i<j}\left[\ln \left|\frac{u_{i}-u_{j}}{2 L / M}\right|+\ln \left|\frac{v_{i}-v_{j}}{2 L / M}\right|\right]-2 \sum_{i, j} \ln \left|\frac{u_{i}-v_{j}}{2 L / M}\right|\right\} \\
& =\left(\frac{2 L}{M}\right)^{2 N}\left|\frac{\prod_{i<j}\left(u_{i}-u_{j}\right)\left(v_{i}-v_{j}\right)}{\prod_{i, j}\left(u_{i}-v_{j}\right)}\right|^{2} \\
& =\left(\frac{2 L}{M}\right)^{2 N}\left|\operatorname{det}\left(\frac{1}{u_{i}-v_{j}}\right)_{(i, j) \in\{1, \ldots, N\}^{2}}\right|^{2} .
\end{aligned}
$$

where we had to choose the particular case of a coupling constant $\Gamma=\beta q^{2}=2$.

Following Ref. [6], to avoid divergences we start from a discretized model in which two sub-lattices $U$ and $V$ are introduced . The positive (negative) particles sit on the sub-lattice $U(V)$. Each lattice site is occupied by no or one particle. The grand canonical partition function defined as a sum including only neutral systems is then

$$
\Xi=1+\sum_{N=1}^{\infty}\left(\frac{2 L}{M}\right)^{2 N} \lambda_{-}^{N} \lambda_{+}^{N} \sum_{\substack{u_{1}, \ldots, u_{N} \in U \\ v_{1}, \ldots, v_{N} \in V}}\left|\operatorname{det}\left(\frac{1}{u_{i}-v_{j}}\right)_{(i, j) \in\{1, \ldots, N\}^{2}}\right|^{2}
$$

where the sums are defined with the prescription that configurations which differ only by a permutation of identical particles are counted only once, and $\lambda_{p}$ is the constant fugacity of the charges of species $p= \pm$. This grand partition function is the determinant of an anti Hermitian matrix $\mathbf{M}$ explicitly shown in Ref. [7].

When passing to the continuum limit in the element $\mathbf{M}_{i j}$ one should replace $u_{i}$ or $v_{i}$ by $z$ and $u_{j}$ or $v_{j}$ by $z^{\prime}$, i.e. $i \rightarrow z$ and $j \rightarrow z^{\prime}$. Each lattice site is characterized by its complex coordinate $z$ and an isospinor which is $\left(\begin{array}{l}1 \\ 0\end{array}\right)$ if the site belongs to the positive sub-lattice $U$ and $\left(\begin{array}{l}0 \\ 1\end{array}\right)$ if it belongs to the negative sub-lattice $V$. We then define a matrix $\mathcal{M}$ by

$$
\left\langle z|\mathcal{M}| z^{\prime}\right\rangle=\frac{\boldsymbol{\sigma}_{\boldsymbol{x}}+i \boldsymbol{\sigma}_{\boldsymbol{y}}}{2} \frac{2 L / M}{z-z^{\prime}}+\frac{\boldsymbol{\sigma}_{\boldsymbol{x}}-i \boldsymbol{\sigma}_{\boldsymbol{y}}}{2} \frac{2 L / M}{\bar{z}-\bar{z}^{\prime}},
$$

where the $\boldsymbol{\sigma}^{\text {'s }}$ are the $2 \times 2$ Pauli matrices operating in the isospinor space, and $z=x+i y$.

The matrix $\mathcal{M}$ can be expressed in terms of a simple Dirac operator

$$
\left\langle z|\mathcal{M}| z^{\prime}\right\rangle=\frac{2 L}{M}\left(\boldsymbol{\sigma}_{\boldsymbol{x}} \partial_{x}+\boldsymbol{\sigma}_{\boldsymbol{y}} \partial_{y}\right) \ln \left|z-z^{\prime}\right|,
$$


and the grand partition function can be rewritten as

$$
\begin{aligned}
\Xi & =\operatorname{det}\left\{\mathbf{1} \delta^{(2)}\left(z ; z^{\prime}\right)+\left[\lambda_{+} \frac{\mathbf{1}+\boldsymbol{\sigma}_{\boldsymbol{z}}}{2}+\lambda_{-} \frac{\mathbf{1}-\boldsymbol{\sigma}_{\boldsymbol{z}}}{2}\right]\left\langle z|\mathcal{M}| z^{\prime}\right\rangle\right\} \\
& =\operatorname{det}\left[\mathbf{I}+\mathbf{K}^{-1}\right]
\end{aligned}
$$

with $\mathbf{I}=\mathbf{1} \delta^{(2)}\left(z ; z^{\prime}\right)$ and

$$
\begin{aligned}
\lambda & =\lambda_{+} \frac{1+\boldsymbol{\sigma}_{\boldsymbol{z}}}{2}+\lambda_{-} \frac{\mathbf{1}-\boldsymbol{\sigma}_{\boldsymbol{z}}}{2}, \\
\mathbf{K}^{-1} & =\boldsymbol{\lambda} \mathcal{M} .
\end{aligned}
$$

Then, since $\Delta_{\text {flat }} \ln |z|=2 \pi \delta(s) \delta(\varphi) / s=2 \pi \delta(z)$ (where $s=|z|$ and $\delta(z)$ is the flat Dirac delta function), the inverse operator $\mathbf{K}$ is

$$
\mathbf{K}=\mathbf{m}^{-1}(z) \mathbf{O}
$$

where

$$
\begin{aligned}
& \mathbf{m}(z)=m_{+}(z) \frac{\mathbf{1}+\boldsymbol{\sigma}_{\boldsymbol{z}}}{2}+m_{-}(z) \frac{\mathbf{1}-\boldsymbol{\sigma}_{\boldsymbol{z}}}{2} \\
& \mathbf{\mathrm { O }}=\frac{2}{M}\left(\boldsymbol{\sigma}_{\boldsymbol{x}} \partial_{x}+\boldsymbol{\sigma}_{\boldsymbol{y}} \partial_{y}\right) .
\end{aligned}
$$

Here $m_{ \pm}(z)=\left(2 \pi L \lambda_{ \pm} \sqrt{g} / s S\right)(2 / M)^{2}$ are rescaled position dependent fugacities, $g=\operatorname{det}\left(g_{\mu \nu}\right)$, and $S$ is the area per lattice site which appears when the discrete sums are replaced by integrals. Notice that in the flat limit $M \rightarrow 0$ we find $m_{ \pm} \rightarrow \widetilde{m}_{ \pm}$, where $\widetilde{m}_{ \pm}=2 \pi L \lambda_{ \pm} / S$ are the rescaled fugacities of the flat system [7, 6]. Moreover $S$ is a local property of the surface independent from its curvature.

We then find

$$
\ln \Xi=\operatorname{Tr}\left\{\ln \left[\mathbf{I}+\mathbf{K}^{-1}\right]\right\},
$$

and the one-body densities and $n$-body truncated densities can be obtained in the usual way by taking functional derivatives of the logarithm of the grand partition function with respect to the fugacities $\lambda_{ \pm}$. Marking the sign of the particle charge at $z_{i}$ by an index $p_{i}= \pm 1$, and defining the matrix

$$
R_{p_{1} p_{2}}\left(z_{1}, z_{2}\right)=\left\langle z_{1} p_{1}\left|\mathbf{K}^{-1}\left(\mathbf{I}+\mathbf{K}^{-1}\right)^{-1}\right| z_{2} p_{2}\right\rangle
$$

it can then be shown [6, 7] that they are given by

$$
\begin{aligned}
& \rho_{p_{1}}^{(1)}\left(z_{1}\right)=R_{p_{1} p_{1}}\left(z_{1}, z_{1}\right), \\
& \rho_{p_{1} p_{2}}^{(2) T}\left(z_{1}, z_{2}\right)=-R_{p_{1} p_{2}}\left(z_{1}, z_{2}\right) R_{p_{2} p_{1}}\left(z_{2}, z_{1}\right), \\
& \rho_{p_{1} p_{2}, \ldots, p_{n}}^{(n) T}\left(z_{1}, z_{2}, \ldots, z_{n}\right)= \\
& (-)^{n+1} \sum_{\left(i_{1}, i_{2}, \ldots, i_{n}\right)} R_{p_{i_{1}} p_{i_{2}}}\left(z_{i_{1}}, z_{i_{2}}\right) \cdots R_{p_{i_{n}} p_{i_{1}}}\left(z_{i_{n}}, z_{i_{1}}\right),
\end{aligned}
$$

where $m_{p}(z)=\left(2 \pi L \lambda_{p} \sqrt{g} / s S\right)(2 / M)^{2}=\left(\widetilde{m}_{p} \sqrt{g} / s\right)(2 / M)^{2}, \widetilde{m}_{p}=2 \pi L \lambda_{p} / S, \sqrt{g}=$ $(M / 2)^{2} s(1+1 / s)^{4}$, and the summation runs over all cycles $\left(i_{1}, i_{2}, \ldots, i_{n}\right)$ built with $\{1,2, \ldots, n\}$. 


\subsection{Symmetries of the Green's function $R$}

Since $\mathbf{m}^{\dagger}(z)=\mathbf{m}(z)$ and $\mathbf{O}^{\dagger}=-\mathbf{O}$ we find

$$
\overline{R_{p_{1} p_{2}}\left(z_{1}, z_{2}\right)}=\left\langle z_{2} p_{2}\left|\mathbf{m}^{-1} \mathbf{O}\left(\mathbf{I}-\mathbf{m}^{-1} \mathbf{O}\right)^{-1}\left(\mathbf{m}^{-1} \mathbf{O}\right)^{-1}\right| z_{1} p_{1}\right\rangle \text {. }
$$

Expanding in $\mathbf{O}$ (by using $\left.\left(\mathbf{I}-\mathbf{m}^{-1} \mathbf{O}\right)^{-1}=\mathbf{I}+\mathbf{m}^{-1} \mathbf{O}+\left(\mathbf{m}^{-1} \mathbf{O}\right)^{2}+\ldots\right)$ and comparing with the definition $R_{p_{1} p_{2}}\left(z_{1}, z_{2}\right)=\left\langle z_{1} p_{1}\left|\left(\mathbf{m}^{-1} \mathbf{O}\right)^{-1}\left(\mathbf{I}+\mathbf{m}^{-1} \mathbf{O}\right)^{-1} \mathbf{m}^{-1} \mathbf{O}\right| z_{2} p_{2}\right\rangle$ we find

$$
\begin{aligned}
& \overline{R_{p p}\left(z_{1}, z_{2}\right)}=R_{p p}\left(z_{2}, z_{1}\right), \\
& \overline{R_{p-p}\left(z_{1}, z_{2}\right)}=-R_{-p p}\left(z_{2}, z_{1}\right) .
\end{aligned}
$$

From which also follows that $R_{p p}\left(z_{1}, z_{1}\right)$ has to be real. If $\lambda_{+}=\lambda_{-}$then we additionally must have

$$
R_{p p}\left(z_{1}, z_{2}\right)=R_{-p-p}\left(z_{1}, z_{2}\right)
$$

\subsection{Two-body truncated correlation functions and perfect screening sum rule}

For the two-body truncated correlation functions of Eq. (35) we then find

$$
\begin{aligned}
& \rho_{++}^{(2) T}\left(z_{1}, z_{2}\right)=-\left|R_{++}\left(z_{1}, z_{2}\right)\right|^{2}, \\
& \rho_{+-}^{(2) T}\left(z_{1}, z_{2}\right)=\left|R_{+-}\left(z_{1}, z_{2}\right)\right|^{2} .
\end{aligned}
$$

Notice that the total correlation function for the like particles $h_{++}\left(z_{1}, z_{2}\right)=$ $\rho_{++}^{(2) T}\left(z_{1}, z_{2}\right) / \rho_{+}^{(1)}\left(z_{1}\right) \rho_{+}^{(1)}\left(z_{2}\right)$ goes to -1 when the particles coincide $z_{1} \rightarrow z_{2}$ as follows from the structure of Eqs. (34)-(35). Moreover the truncated densities of any order has to decay to zero as two groups of particles are infinitely separated. In particular $\left|R_{++}\left(z_{1}, z_{2}\right)\right|=\left|R_{++}(s 1, s 2 ; \varphi)\right|$, with $\varphi=\varphi_{1}-\varphi_{2}$, has to decay to zero as $\left|s_{1}-s_{2}\right| \rightarrow \infty$.

The perfect screening sum rule has to be satisfied for the symmetric mixture

$$
\int\left[\rho_{+-}^{(2) T}\left(z_{1}, z_{2}\right)-\rho_{++}^{(2) T}\left(z_{1}, z_{2}\right)\right] \sqrt{g_{1}} d s_{1} d \varphi_{1}=\rho_{ \pm}\left(z_{2}\right)
$$

where $g_{1}$ is $g$ calculated on particle 1 .

\subsection{From the structure to the thermodynamics}

Following Ref. [12, we note that if we solve the eigenvalue problem $\mathbf{K} \psi_{n}=\lambda_{n} \psi_{n}$ (here $\psi_{n}$ is a spinor of components $\psi_{+, n}$ and $\psi_{-, n}$ ), then

$$
\Xi=\prod_{n}\left(1+\frac{1}{\lambda_{n}}\right)
$$

Now the eigenvalue problem reads

$$
\begin{aligned}
& \left\{m_{+}(z)+A\left[m_{-}(z)\right]^{-1} A^{\dagger}\right\} \psi_{-}(z)=\left(m_{-}(z)-\lambda^{2}\right) \psi_{-}(z), \\
& \left\{m_{-}(z)+A^{\dagger}\left[m_{+}(z)\right]^{-1} A\right\} \psi_{+}(z)=\left(m_{-}(z)-\lambda^{2}\right) \psi_{+}(z),
\end{aligned}
$$

where $A=(2 / M)\left(\partial_{x}+i \partial_{y}\right)$ and $A^{\dagger}=(2 / M)\left(-\partial_{x}+i \partial_{y}\right)$ and the left hand side is the same as the left hand side for the Green's functions Eqs. (49)-(150).

Of course we know that the thermodynamic quantities all diverge in our $\Gamma=2$ case so Eq. (44), although suggestive, is of small practical interest. 


\subsection{Determination of the Green's function $R$}

The Green's function matrix $\mathbf{R}$ is the solution of a system of four coupled partial differential equations, namely

$$
\left(\mathbf{I}+\mathbf{K}^{-1}\right) \mathbf{K R}\left(z_{1}, z_{2}\right)=(\mathbf{I}+\mathbf{K}) \mathbf{R}\left(z_{1}, z_{2}\right)=\mathbf{1} \delta^{(2)}\left(z_{1} ; z_{2}\right)
$$

where $\delta^{(2)}\left(z_{1} ; z_{2}\right)=\delta^{(2)}\left(s, \varphi ; s_{0}, \varphi_{0}\right)=(\sqrt{g})^{-1} \delta\left(s-s_{0}\right) \delta\left(\varphi-\varphi_{0}\right)$ is the Dirac delta function on the curved surface, $\delta\left(s-s_{0}\right) \delta\left(\varphi-\varphi_{0}\right) / s=\delta\left(s, \varphi ; s_{0}, \varphi_{0}\right)=\delta\left(z ; z_{0}\right)$ is the flat Dirac delta function, and 1 is the identity matrix. These can be rewritten as follows

$$
\left[\mathbf{O}+\mathbf{m}\left(z_{1}\right)\right] \mathbf{R}\left(z_{1}, z_{2}\right)=\mathbf{m}\left(z_{1}\right) \delta^{(2)}\left(z_{1} ; z_{2}\right) .
$$

If instead of $\mathbf{R}$ one uses $\mathbf{R}=\mathbf{G} \widetilde{\mathbf{m}}, \mathbf{G}$ satisfies the equation

$$
\left[\mathbf{O}+\mathbf{m}\left(z_{1}\right)\right] \mathbf{G}\left(z_{1}, z_{2}\right)=\frac{4}{M^{2}} \mathbf{1} \delta\left(z_{1} ; z_{2}\right),
$$

where $\delta\left(z_{1} ; z_{2}\right)$ is now the flat Dirac delta function [18].

By combining the components of this equation one obtains decoupled equations for $G_{++}$and $G_{--}$as follows

$$
\begin{aligned}
\left\{m_{+}\left(z_{1}\right)+A^{\dagger}\left[m_{-}\left(z_{1}\right)\right]^{-1} A\right\} G_{++}\left(z_{1}, z_{2}\right) & =\frac{4}{M^{2}} \delta\left(s_{1}, \varphi_{1} ; s_{2}, \varphi_{2}\right), \\
\left\{m_{-}\left(z_{1}\right)+A\left[m_{+}\left(z_{1}\right)\right]^{-1} A^{\dagger}\right\} G_{--}\left(z_{1}, z_{2}\right) & =\frac{4}{M^{2}} \delta\left(s_{1}, \varphi_{1} ; s_{2}, \varphi_{2}\right),
\end{aligned}
$$

while

$$
\begin{aligned}
& G_{-+}\left(z_{1}, z_{2}\right)=-\left[m_{-}\left(z_{1}\right)\right]^{-1} A G_{++}\left(z_{1}, z_{2}\right), \\
& G_{+-}\left(z_{1}, z_{2}\right)=+\left[m_{+}\left(z_{1}\right)\right]^{-1} A^{\dagger} G_{--}\left(z_{1}, z_{2}\right),
\end{aligned}
$$

Then Eq. (49) can be rewritten in Cartesian coordinates as

$$
\begin{aligned}
& \left\{m_{+}\left(z_{1}\right) m_{-}\left(z_{1}\right)-\left(\frac{2}{M}\right)^{2}\left[\left(\partial_{x_{1}}^{2}+\partial_{y_{1}}^{2}\right)-\right.\right. \\
& \left.\left.\frac{4\left(-x_{1}+i y_{1}\right)}{s_{1}^{2}\left(1+s_{1}\right)}\left(\partial_{x_{1}}+i \partial_{y_{1}}\right)\right]\right\} G_{++}\left(z_{1}, z_{2}\right)= \\
& \left(\frac{2}{M}\right)^{4} \frac{\tilde{m}_{-\sqrt{g_{1}}}}{s_{1}^{2}} \delta\left(s_{1}-s_{2}\right) \delta\left(\varphi_{1}-\varphi_{2}\right)= \\
& \left(\frac{2}{M}\right)^{4} \frac{\widetilde{m}_{-\sqrt{g_{1}}}}{\sqrt{x_{1}^{2}+y_{1}^{2}}} \delta\left(x_{1}-x_{2}\right) \delta\left(y_{1}-y_{2}\right),
\end{aligned}
$$

where $s=\sqrt{x^{2}+y^{2}}$. From the expression of the gradient in polar coordinates follows

$$
\left\{\begin{array}{l}
\partial_{x}=\cos \varphi \partial_{s}-\frac{\sin \varphi}{{ }_{s}} \partial_{\varphi} \\
\partial_{y}=\sin \varphi \partial_{s}+\frac{\cos \varphi}{s} \partial_{\varphi}
\end{array}\right.
$$


Which allows us to rewrite Eq. (53) in polar coordinates as

$$
\begin{aligned}
& {\left[\widetilde{m}_{+} \widetilde{m}_{-}\left(1+\frac{1}{s_{1}}\right)^{8}-\left(\frac{2}{M}\right)^{2}\left(\frac{1}{s_{1}} \partial_{s_{1}}\left(s_{1} \partial_{s_{1}}\right)+\frac{1}{s_{1}^{2}} \partial_{\varphi_{1}}^{2}+\right.\right.} \\
& \left.\left.\frac{4}{s_{1}\left(1+s_{1}\right)} \partial_{s_{1}}+\frac{4 i}{s_{1}^{2}\left(1+s_{1}\right)} \partial_{\varphi_{1}}\right)\right] G_{++}\left(z_{1}, z_{2}\right)= \\
& \left(\frac{2}{M}\right)^{4} \frac{\widetilde{m}_{-} \sqrt{g_{1}}}{s_{1}^{2}} \delta\left(s_{1}-s_{2}\right) \delta\left(\varphi_{1}-\varphi_{2}\right) .
\end{aligned}
$$

From this equation we immediately see that $G_{++}\left(z_{1}, z_{2}\right)$ cannot be real. Notice that in the flat limit $M \rightarrow 0$ we have $s \sim 2 r / M$ and Eq. (55) reduces to

$$
\begin{aligned}
& {\left[\widetilde{m}_{+} \widetilde{m}_{-}-\frac{1}{r_{1}} \partial_{r_{1}}\left(r_{1} \partial_{r_{1}}\right)-\frac{1}{r_{1}^{2}} \partial_{\varphi_{1}}^{2}\right] G_{++}\left(z_{1}, z_{2}\right)=} \\
& \frac{\widetilde{m}_{-}}{r_{1}} \delta\left(r_{1}-r_{2}\right) \delta\left(\varphi_{1}-\varphi_{2}\right) .
\end{aligned}
$$

which, when $\widetilde{m}_{+}=\widetilde{m}_{-}=\widetilde{m}$, has the following well known solution [7, 6]

$$
G_{++}\left(z_{1}, z_{2}\right)=\frac{\widetilde{m}}{2 \pi} K_{0}\left(\widetilde{m}\left|\mathbf{r}_{1}-\mathbf{r}_{2}\right|\right)
$$

where $K_{0}$ is a modified Bessel function.

Let us from now on restrict to the case of equal fugacities of the two species. Then $\lambda_{-}=\lambda_{+}=\lambda$ with

$$
\widetilde{m}=\frac{2 \pi L}{S} \lambda=\frac{2 \pi L e^{\beta \mu}}{\Lambda^{2}}=\left(2 \pi L \frac{m q^{2}}{4 \pi \hbar^{2}}\right) e^{2 \mu / q^{2}},
$$

where $\hbar$ is Planck's constant, $m$ is the mass of the particles, and $\mu$ the chemical potential. So $\widetilde{m}$ has the dimensions of an inverse length. From the symmetry of the problem we can say that $G_{++}=G_{++}\left(s_{1}, s_{2} ; \varphi_{1}-\varphi_{2}\right)$. We can then express the Green's function as the following Fourier series expansion

$$
G_{++}\left(s_{1}, s_{2} ; \varphi\right)=\frac{1}{2 \pi} \sum_{k=-\infty}^{\infty} g_{++}\left(s_{1}, s_{2} ; k\right) e^{i k \varphi} .
$$

Then, using the expansion of the Dirac delta function, $\sum_{k} e^{i k \varphi}=2 \pi \delta(\varphi)$, we find that $g_{++}$, a continuous real function symmetric under exchange of $s_{1}$ and $s_{2}$, has to satisfy the following equation

$$
\begin{aligned}
& {\left[Q_{0}\left(k, s_{1}\right)+Q_{1}\left(s_{1}\right) \partial_{s_{1}}+Q_{2}\left(s_{1}\right) \partial_{s_{1}}^{2}\right] g_{++}\left(s_{1}, s_{2} ; k\right)=} \\
& \left(\frac{2}{M}\right)^{2} \widetilde{m} s_{1}^{3}\left(1+s_{1}\right)^{5} \delta\left(s_{1}-s_{2}\right),
\end{aligned}
$$

where

$$
\begin{aligned}
Q_{0}(k, s) & =\widetilde{m}^{2}(1+s)^{9}+\left(\frac{2}{M}\right)^{2} k s^{6}(4+k(1+s)) \\
Q_{1}(s) & =-\left(\frac{2}{M}\right)^{2} s^{7}(5+s) \\
Q_{2}(s) & =-\left(\frac{2}{M}\right)^{2} s^{8}(1+s) .
\end{aligned}
$$


And the coefficients $Q_{i}$ are polynomials of up to degree 9 .

\subsection{Method of solution}

We start from the homogeneous form of Eq. (60). We note that, for a given $k$, the two linearly independent solutions $f_{\alpha}(s ; k)$ and $f_{\beta}(s ; k)$ of this linear homogeneous second order ordinary differential equation are not available in the mathematical literature to the best of our knowledge. Assuming we knew those solutions we would then find the Green's function, $g_{++}\left(s_{1}, s_{2} ; k\right)$, writing [19]

$$
f\left(t_{1}, t_{2} ; k\right)=c_{k} f_{\alpha}\left(s_{<} ; k\right) f_{\beta}\left(s_{>} ; k\right) \text {, }
$$

where $s_{<}=\min \left(s_{1}, s_{2}\right), s_{>}=\max \left(s_{1}, s_{2}\right)$, and $f_{\beta}$ has the correct behavior at large $s$. Then we determine $c_{k}$ by imposing the kink in $f$ due to the Dirac delta function at $s_{1}=s_{2}$ as follows

$$
\left.\partial_{s_{1}} f\left(s_{1}, s_{2} ; k\right)\right|_{s_{1}=s_{2}+\epsilon}-\left.\partial_{s_{1}} f\left(s_{1}, s_{2} ; k\right)\right|_{s_{1}=s_{2}-\epsilon}=-\widetilde{m} \frac{\left(1+s_{2}\right)^{4}}{s_{2}^{5}},
$$

where $\epsilon$ is small and positive.

The Green's function, symmetric under exchange of $s_{1}$ and $s_{2}$, is reconstructed as follows

$$
G_{++}\left(z_{1}, z_{2}\right)=G_{++}\left(s_{1}, s_{2} ; \varphi\right)=\frac{1}{2 \pi} \sum_{k=-\infty}^{\infty} c_{k} f_{\alpha}\left(s_{<} ; k\right) f_{\beta}\left(s_{>} ; k\right) e^{i k \varphi}
$$

\section{TCP in the whole surface}

On the whole surface, using Eq. (21), we can now write $e^{-\beta U_{2 N}}$ at a coupling constant $\Gamma=2$ as follows

$$
\left|\operatorname{det}\left(\frac{L}{L_{0}} \frac{\sqrt{\left|u_{j} v_{j}\right|}}{u_{i}-v_{j}}\right)_{(i, j) \in\{1, \ldots, N\}^{2}}\right|^{2} .
$$

The grand partition function will then be

$$
\Xi=\operatorname{det}\left[\mathbf{I}+\mathbf{K}_{\mathrm{WS}}{ }^{-1}\right] \text {, }
$$

with

$$
\begin{aligned}
\mathbf{K}_{\mathrm{WS}}{ }^{-1} & =\left(\frac{M}{2 L_{0}}\right) \lambda_{\mathrm{WS}} \mathcal{M}, \\
\lambda_{\mathrm{WS}} & =\lambda_{+}|z| \frac{\mathbf{1}+\boldsymbol{\sigma}_{\boldsymbol{z}}}{2}+\lambda_{-}|z| \frac{\mathbf{1}-\boldsymbol{\sigma}_{\boldsymbol{z}}}{2} .
\end{aligned}
$$

The inverse operator is then given by

$$
\begin{aligned}
\mathbf{K}_{\mathrm{WS}} & =\left(\frac{2 L_{0}}{M}\right) \mathcal{M}^{-1} \lambda_{\mathrm{WS}}^{-1} \\
\lambda_{\mathrm{WS}}^{-1} & =\frac{1}{\lambda_{+}|z|} \frac{\mathbf{1}+\boldsymbol{\sigma}_{\boldsymbol{z}}}{2}+\frac{1}{\lambda_{-}|z|} \frac{\mathbf{1}-\boldsymbol{\sigma}_{\boldsymbol{z}}}{2}, \\
\mathcal{M}^{-1} & =\frac{S s}{2 \pi L \sqrt{g}}\left(\frac{M}{2}\right)^{2} \mathrm{O} .
\end{aligned}
$$


Two Component Plasma in a Flamm's Paraboloid

Introducing position dependent fugacities [20]

$$
m_{p}(z)=\frac{2 \pi\left(L / L_{0}\right) \lambda_{p} \sqrt{g}}{S s}=\widetilde{m}_{p} \frac{\sqrt{g}}{s}
$$

where now $\widetilde{m}_{p} / L_{0} \rightarrow \widetilde{m}_{p}$, we can rewrite

$$
\mathbf{K}_{\mathrm{WS}}=\frac{\boldsymbol{\sigma}_{\boldsymbol{x}}+i \boldsymbol{\sigma}_{\boldsymbol{y}}}{2} a_{-}+\frac{\boldsymbol{\sigma}_{\boldsymbol{x}}-i \boldsymbol{\sigma}_{\boldsymbol{y}}}{2} a_{+}
$$

with the operators

$$
\begin{aligned}
& a_{-}=-\frac{\bar{z}}{m_{-}(z)|z|^{3}}+\frac{1}{m_{-}(z)|z|}\left(\partial_{x}-i \partial_{y}\right), \\
& a_{+}=-\frac{z}{m_{+}(z)|z|^{3}}+\frac{1}{m_{+}(z)|z|}\left(\partial_{x}+i \partial_{y}\right) .
\end{aligned}
$$

Then the equation for the Green's functions are

$$
\begin{aligned}
& \left(1-a_{-} a_{+}\right) R_{++}\left(z_{1}, z_{2}\right)=\delta^{(2)}\left(z_{1} ; z_{2}\right) \\
& \left(1-a_{+} a_{-}\right) R_{--}\left(z_{1}, z_{2}\right)=\delta^{(2)}\left(z_{1} ; z_{2}\right), \\
& R_{+-}=-a_{-} R_{--}, \\
& R_{-+}=-a_{+} R_{++} .
\end{aligned}
$$

The equation for $R_{++}$in the symmetric mixture case is

$$
\begin{aligned}
& {\left[m^{2}\left(z_{1}\right)-\frac{2}{s_{1}^{4}}+\frac{2 \partial_{s_{1}}}{s_{1}^{3}}-\frac{\partial_{s_{1}}^{2}}{s_{1}^{2}}-\frac{-i \partial_{\varphi_{1}}+\partial_{\varphi_{1}}^{2}}{s_{1}^{4}}\right] R_{++}\left(z_{1}, z_{2}\right)=} \\
& \frac{m^{2}\left(z_{1}\right)}{\sqrt{g_{1}}} \delta\left(s_{1}-s_{2}\right) \delta\left(\varphi_{1}-\varphi_{2}\right)=\frac{\tilde{m}^{2} \sqrt{g_{1}}}{s_{1}^{2}} \delta\left(s_{1}-s_{2}\right) \delta\left(\varphi_{1}-\varphi_{2}\right)
\end{aligned}
$$

From this equation we see that $R_{++}\left(z_{1}, z_{2}\right)$ will now be real.

By expanding Eq. (79) in a Fourier series in the azimuthal angle we now find

$$
\begin{aligned}
& {\left[Q_{0}\left(k, s_{1}\right)+Q_{1}\left(s_{1}\right) \partial_{s_{1}}+Q_{2}\left(s_{1}\right) \partial_{s_{1}}^{2}\right] g_{++}\left(s_{1}, s_{2} ; k\right)=} \\
& \left(\frac{M}{2}\right)^{2} \widetilde{m} s_{1}^{3}\left(1+s_{1}\right)^{4} \delta\left(s_{1}-s_{2}\right),
\end{aligned}
$$

where

$$
\begin{aligned}
& Q_{0}(k, s)=\left(\frac{M}{2}\right)^{4} \tilde{m}^{2}(1+s)^{8}+s^{4}\left(k^{2}-k-2\right) \\
& Q_{1}(s)=2 s^{5} \\
& Q_{2}(s)=-s^{6} .
\end{aligned}
$$

And the coefficients $Q_{i}$ are now polynomials of up to degree 8 .

In the flat limit we find, for $G_{++}=R_{++} / \widetilde{m}$, the following equation

$$
\begin{aligned}
& {\left[\widetilde{m}^{2}-\frac{2}{r_{1}^{4}}+\frac{2 \partial_{r_{1}}}{r_{1}^{3}}-\frac{\partial_{r_{1}}^{2}}{r_{1}^{2}}-\frac{-i \partial_{\varphi_{1}}+\partial_{\varphi_{1}}^{2}}{r_{1}^{4}}\right] G_{++}\left(z_{1}, z_{2}\right)=} \\
& \frac{\widetilde{m}}{r_{1}} \delta\left(r_{1}-r_{2}\right) \delta\left(\varphi_{1}-\varphi_{2}\right) .
\end{aligned}
$$


We then see that we now do not recover the TCP in the plane [7, 6]. This has to be expected because in the flat limit the Flamm's paraboloid reduces to two planes connected by the origin.

After the Fourier expansion of Eq. (59) we now get

$$
\left[P_{0}\left(k, r_{1}\right)+P_{1}\left(r_{1}\right) \partial_{r_{1}}+P_{2}\left(r_{1}\right) \partial_{r_{1}}^{2}\right] g_{++}\left(r_{1}, r_{2} ; k\right)=\widetilde{m} \delta\left(r_{1}-r_{2}\right)
$$

where

$$
\begin{aligned}
& P_{0}(k, r)=\tilde{m}^{2} r+\frac{k^{2}-k-2}{r^{3}}, \\
& P_{1}(r)=\frac{2}{r^{2}}, \\
& P_{2}(r)=-\frac{1}{r} .
\end{aligned}
$$

The homogeneous form of this equation admits the following two linearly independent solutions

$$
\begin{aligned}
& \left.\begin{array}{l}
f_{1}(r ;-1)=\left[D_{-1 / 2}(i \sqrt{2 \widetilde{m}} r)+\overline{D_{-1 / 2}(i \sqrt{2 \widetilde{m}} r)}\right] / 2 \\
f_{2}(r ;-1)=D_{-1 / 2}(\sqrt{2 \widetilde{m}} r)
\end{array}\right\} \quad k=-1,
\end{aligned}
$$

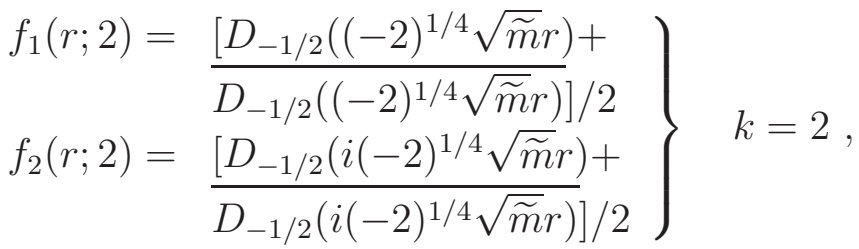

$$
\begin{aligned}
& \left.\begin{array}{l}
f_{1}(t ; k)=\sqrt{r} I_{-\sqrt{7-4 k+4 k^{2}} / 4}\left(\tilde{m} r^{2} / 2\right) \\
f_{2}(t ; k)=\sqrt{r} I_{\sqrt{7-4 k+4 k^{2}} / 4}\left(\tilde{m} r^{2} / 2\right)
\end{array}\right\} \quad \text { else },
\end{aligned}
$$

where $D_{\nu}(x)$ are parabolic cylinder functions and $I_{\mu}(x)$ are the modified Bessel functions of the first kind which diverge as $e^{x} / \sqrt{2 \pi x}$ for large $x \gg\left|\mu^{2}-1 / 4\right|$.

Again we write $g_{++}\left(r_{1}, r_{2} ; k\right)=c_{k} f_{\alpha}\left(r_{<} ; k\right) f_{\beta}\left(r_{>} ; k\right)$ and impose the kink condition,

$$
\left.\partial_{r_{1}} g_{++}\left(r_{1}, r_{2} ; k\right)\right|_{r_{1}=r_{2}+\epsilon}-\left.\partial_{r_{1}} g_{++}\left(r_{1}, r_{2} ; k\right)\right|_{r_{1}=r_{2}-\epsilon}=-\widetilde{m} r_{2}
$$

to find the $c_{k}$. The Green's function is then reconstructed using Eq. (63). But we immediately see that curiously $\left|G_{++}\right|$diverges. Even the structure of the plasma is not well defined in this situation. The collapse of opposite charges at the horizon shrinking to the origin makes the structure of the plasma physically meaningless.

\section{Conclusions}

An attempt was made to extend the solution of the one component plasma, at the special value of the coupling constant $\Gamma=2$, living in the Flamm's paraboloid [16] to the two component one, the TCP. The Flamm's paraboloid is a Riemannian surface with non-constant curvature which is asymptotically flat. Its curvature depends on a parameter $M$ ( $2 M$ being the radius of the horizon) in such way that for $M \rightarrow 0$ the surface becomes flat: two planes connected by the origin. 
The work of F. Cornu and B. Jancovici [7, 6] on the TCP in a plane showed that at such particular value of the coupling constant, using a Cauchy's identity, it is possible to find an analytic solution to the structure of the plasma starting from a discretized model to avoid divergences and taking the continuous limit in the end. All the $n$-particle correlation functions with $n>1$ are well behaved except the particle density which diverges as all the other thermodynamic quantities due to the competition between the attraction of oppositely charged particles and the thermal motion. This can be prevented by adding an hard core to the particles in order to prevent the collapse.

In this work we repeated the same calculation for the plasma living in the Flamm's paraboloid, in the half surface case, $s>1$, with an insulating horizon (at $s=1$ ) and in the full surface case, $s>0$. The solution of the equation determining the structure, the Green's function equation, is reduced to the mathematical problem of finding the two linearly independent solutions of a linear homogeneous second order ordinary differential equation with polynomial coefficients of high degree (9th in the half surface case and 8th in the full surface case). To the best of our knowledge, an analytic solution of the equations turns out to be out of mathematical reach. Nonetheless the many-body problem has been reduced to finding the solutions of a simple differential equation. The further step of finding explicitly the alanytical form of the solutions from the given differential equations is a matter of mathematical syntax and we think does not add much to the present work.

We discussed the symmetries of the Green's function, the perfect screening sum rule, the relationship between the structure and the thermodynamics, and the method of solution of the Green's function equation.

We found that for the plasma living in the half Flamm's paraboloid with an insulating horizon the flat limit $M \rightarrow 0$ reduces the system to the TCP on the plane [7, 6]. For the plasma living in the full Flamm's paraboloid taking the flat limit one does not recover the plasma in the plane and this has to be expected since the paraboloid in this limit reduces to two planes connected by a hole at the origin. Instead the resulting solution for the structure turns out to be physically meaningless. The collapse of opposite charges at the horizon as it shrinks to the origin spoils the structure of the plasma.

We leave as an open problem the one of finding approximations to the two linearly independent solutions of the homogeneous counterpart of Eq. (60), for the half surface case, and of Eq. (80), for the whole surface case, as a function of the integer $k$, the further determination of the Green function in these two cases, through Eq. (63), the eventual analysis of the solution and discussion about the necessity of the addition of a hard core to the particles (hard "disks" of diameter $D$ with $\left.\delta s<2 D(1+1 / s)^{-2} / M\right)$ in order to cure the divergence of the densities $\rho_{p}$ with the consequent determination of the equation of state. These final steps are necessary in order to have the problem of the structure of the fluid reduced to a closed form. In the present work we limited ourselves to what can be said in an 
analytical form.

\section{Acknowledgments}

I would like to acknowledge the financial support and hospitality from the National Institute for Theoretical Physics of South Africa during the preparation of the work. I would also like to thank Peter Forrester for stimulating the work and Filippo Giraldi for discussions on the solution of the Green's function equation.

\section{References}

[1] A. Salzberg and S. Prager. J. Chem. Phys., 38:2587, 1963.

[2] E. H. Hauge and P. C. Hemmer. Phys. Norvegica, 5:209, 1971.

[3] L. Šamaj and I. Travěnec. J. Stat. Phys., 101:713, 2000.

[4] J. M. Kosterlitz and D. J. Thouless. J. Phys. C, 6:1181, 1973.

[5] A. Lenard. J. Math. Phys., 2:682, 1961.

[6] F. Cornu and B. Jancovici. J. Stat. Phys., 49:33, 1987.

[7] F. Cornu and B. Jancovici. J. Chem. Phys., 90:2444, 1989.

[8] P. J. Forrester. J. Chem. Phys., 95:4545, 1991.

[9] G. Téllez and L. Merchán. J. Stat. Phys., 108:495, 2002.

[10] L. Merchán and G. Téllez. J. Stat. Phys., 114:735, 2004.

[11] G. Téllez. J. Chem. Phys., 106:8572, 1997.

[12] G. Téllez. J. Phys. A: Mathematical and General, 31:5277, 1998.

[13] P. J. Forrester, B. Jancovici, and J. Madore. J. Stat. Phys., 69:179, 1992.

[14] P. J. Forrester and B. Jancovici. J. Stat. Phys., 84:337, 1996.

[15] B. Jancovici and G. Téllez. J. Stat. Phys., 91:953, 1998.

[16] R. Fantoni and G. Téllez. J. Stat. Phys., 133:449, 2008.

[17] A. C. Aitken. Determinants and Matrices. Interscience, New York, 1956.

[18] The same result could have been reached by noticing that the partition function of Eq. (223) rewritten in the continuum is equivalent to the partition function of the flat system with position dependent fugacities $\zeta_{p}(s)=\lambda_{p}(M / 2)^{2}(1+1 / s)^{4}(2 L / M)$. This leads naturally [7] to define a green function $\tilde{\mathbf{G}}=(M / 2) \mathbf{G}$ which satisfies the equation $[(M / 2) \mathbf{O}+$ $(2 \pi / S) \boldsymbol{\zeta}(z)] \tilde{\mathbf{G}}\left(z, z^{\prime}\right)=\mathbf{1} \delta\left(z ; z^{\prime}\right)$ which reduces to Eq. (48).

[19] J. D. Jackson. Classical Electrodynamics. John Wiley \& Sons, Inc., United States of America, third edition, 1999. Sections $3.9 \& 3.11$.

[20] Alternatively we could have left the one body terms $(\sqrt{|z|})$ outside the determinant, and then consider them as part of the position depend fugacities, and simply apply F. Cornu and B. Jancovici 7 formalism with these position depend fugacities. 